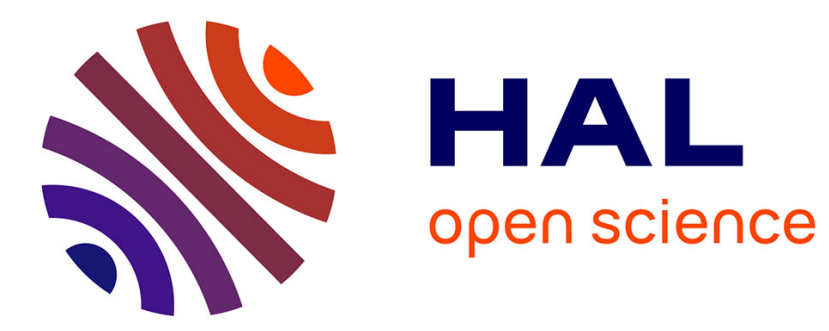

\title{
Estimation de l'effet réservoir 14C marin en mer d'Arabie
}

\author{
Jean-François Saliège, Anne-Marie Lézine, Serge Cleuziou
}

\section{To cite this version:}

Jean-François Saliège, Anne-Marie Lézine, Serge Cleuziou. Estimation de l'effet réservoir 14C marin en mer d'Arabie. Paléorient, 2005, 31 (1), pp.64 - 69. 10.3406/paleo.2005.4784 . hal-01613982

\section{HAL Id: hal-01613982 \\ https://hal.science/hal-01613982}

Submitted on 19 Feb 2021

HAL is a multi-disciplinary open access archive for the deposit and dissemination of scientific research documents, whether they are published or not. The documents may come from teaching and research institutions in France or abroad, or from public or private research centers.
L'archive ouverte pluridisciplinaire HAL, est destinée au dépôt et à la diffusion de documents scientifiques de niveau recherche, publiés ou non, émanant des établissements d'enseignement et de recherche français ou étrangers, des laboratoires publics ou privés.

\section{(1) (1) $\$$}

Distributed under a Creative Commons Attribution - NonCommercial - NoDerivatives| 4.0 


\title{
Estimation de l'effet réservoir 14C marin en mer d'Arabie J.-F. Saliège, Anne-Marie Lezine, S. Cleuziou
}

\section{Résumé}

Les mesures de l'effet réservoir en mer d'Arabie, de l'actuel à 8685 cal BC, permettent d'envisager la calibration 14C des biominéralisations marines (coquilles, otolithes, bioapatite). Du présent à 4560 cal $\mathrm{BC}$, le $\Delta \mathrm{R}$ reste constant ( $210 \pm 75$ ans) dans le nord-ouest de la mer d'Arabie, ce qui permet d'insérer les dates $14 \mathrm{C}$ de nombreux sites anthropiques des rivages du golfe d'Oman dans les chronologies classiques. Au-delà, le $\Delta R$ enregistre les importantes variations d'intensités des remontées d'eau profonde. II atteint jusqu 'à 700 ans de 6475 cal BC à 7305 cal BC.

\begin{abstract}
Measurements of the marine reservoir effect in the Arabian Sea, from the present to 8685 cal BC, make it possible to discuss the $14 \mathrm{C}$ calibration of marine biomineralisations (shells, otoliths, bioapatite). From the present to 4560 cal $\mathrm{BC}$, the $\Delta R(210 \pm 15)$ remains constant in the north-west of Arabian Sea allowing us to insert the 14C dates of many anthropic sites from the coast of the gulf of Oman into classical chronologies. During the early to mid-Holocene, the high amplitude in sea surface temperature variation linked to intensified upwelling activity lead to higher values of the marine reservoir effect of up to 700 years from 6475 to 7305 cal BC.
\end{abstract}

\section{Citer ce document / Cite this document :}

Saliège J.-F., Lezine Anne-Marie, Cleuziou S. Estimation de l'effet réservoir 14C marin en mer d'Arabie. In: Paléorient, 2005, vol. 31, $n^{\circ} 1$. Anciennes exploitations des mers et des cours d'eau en Asie du Sud-Ouest. Approches environnementales. pp. 64-69;

doi : https://doi.org/10.3406/paleo.2005.4784

https://www.persee.fr/doc/paleo_0153-9345_2005_num_31_1_4784

Fichier pdf généré le 27/10/2018 


\title{
ESTIMATION DE L'EFFET RÉSERVOIR ${ }^{14}$ C MARIN EN MER D'ARABIE
}

\author{
J.-F. SALIÈGE, A.-M. LÉZINE et S. CLEUZIOU
}

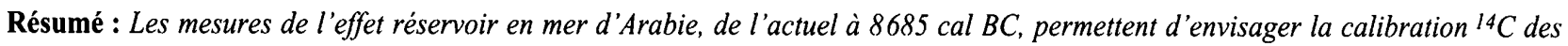
biominéralisations marines (coquilles, otolithes, bioapatite). Du présent à 4560 cal BC, le $\Delta R^{1}$ reste constant $(210 \pm 15$ ans) dans le nord-ouest de la mer d'Arabie, ce qui permet d'insérer les dates ${ }^{14} \mathrm{C}$ de nombreux sites anthropiques des rivages du golfe d'Oman dans les chronologies classiques. Au-delà, le $\Delta R$ enregistre les importantes variations d'intensités des remontées d'eau profonde. Il atteint jusqu'à 700 ans de $6475 \mathrm{cal} B C$ à 7305 cal BC.
\end{abstract}

\begin{abstract}
Measurements of the marine reservoir effect in the Arabian Sea, from the present to 8685 cal BC, make it possible to discuss the ${ }^{14} \mathrm{C}$ calibration of marine biomineralisations (shells, otoliths, bioapatite). From the present to 4560 cal $B C$, the $\Delta R(210 \pm 15)$ remains constant in the north-west of Arabian Sea allowing us to insert the ${ }^{14} \mathrm{C}$ dates of many anthropic sites from the coast of the gulf of Oman into classical chronologies. During the early to mid-Holocene, the high amplitude in sea surface temperature variation linked to intensified upwelling activity lead to higher values of the marine reservoir effect of up to 700 years from 6475 to 7305 cal BC.
\end{abstract}

Mots Clefs : Effet réservoir, ${ }^{14} \mathrm{C}$, Upwelling, $\Delta R$.

Key-Words : Marine reservoir effect, Upwelling activity, $\Delta R$.

La chronologie des sites anthropiques des rivages du golfe d'Oman est fondée essentiellement sur l'étude stylistique de la céramique ${ }^{2}$. Au-delà des périodes historiques, le golfe d'Oman se trouvant alors sur le parcours des grands réseaux commerciaux dominés par les civilisations de Sumer et de l'Indus, les chronologies régionales - reposant sur les céramiques d'importation ou locale - peuvent être raccordées à ces deux grands ensembles de l'Asie moyenne. Cependant pour bien des sites protohistoriques et, pour les périodes antérieures, l'absence de céramiques impose le recours à une solution alternative. Seule une méthode physico-chimique devient envisageable. La datation ${ }^{14} \mathrm{C}$ se révèle la plus précise à la condition de pouvoir mesurer avec exactitude l'effet réservoir marin.

1. $\Delta \mathrm{R}$ : écart par rapport à l'effet réservoir mondial.

2. E.g. CLEUZIOU and TOSI, 1989 ; MÉRY, 1991, 1996.

\section{LA DATATION ${ }^{14} \mathrm{C}$ EN MILIEU MARIN ET CALIBRATION}

Les sites anthropiques holocènes disséminés le long des rivages de la mer d'Arabie présentent fréquemment d'importantes accumulations de coquilles et d'ossements d'animaux marins. Ces vestiges, reflet d'une activité humaine tournée vers la mer, sont a priori susceptibles de fournir un matériau de datation ${ }^{14} \mathrm{C}$. Cependant, en milieu océanique l'équilibre isotopique entre le $\mathrm{CO}_{2}$ atmosphérique (réservoir du ${ }^{14} \mathrm{C}$ ) et le carbone total inorganique dissous (CTID) dans les eaux n'est pas atteint. Le brassage entre les différentes masses d'eau entraîne un appauvrissement en ${ }^{14} \mathrm{C}$. Les organismes vivants dont la source de carbone est le CTID subissent le même déficit. Les échanges entre le dioxyde de carbone atmosphérique et les eaux marines - de surface, de la zone de thermocline ou 
profondes - ont été modélisés ${ }^{3}$. Du modèle des eaux de surface, testé grâce à des coquilles d'âge connu, on en déduit un vieillissement apparent de l'ordre de 400 ans des êtres vivant en milieu marin par rapport à ceux vivant en milieu continental. C'est ce décalage que l'on nomme effet réservoir mondial (R). Ce dernier étant lié à la circulation des masses d'eaux océaniques n'est pas uniforme. En particulier dans les zones d'upwelling, la remontée des eaux profondes entraîne un effet réservoir plus important. On désigne sous le terme $\Delta R$ cet écart par rapport au $R$ mondial. Ce ne sera qu'une fois $\Delta R$ connu et en respectant les conventions de normalisation des âges ${ }^{14} \mathrm{C}^{4}$ que l'on pourra envisager l'utilisation des logiciels de calibration ${ }^{5}$, qui permettront d'insérer ces dates dans les chronologies historiques.

Pour obtenir une mesure fiable du $\Delta \mathrm{R}$, la solution la plus simple est de connaître l'âge réel de la coquille et d'en effectuer la datation. Les explosions thermonucléaires des années 1950-1963 AD ayant bouleversé la teneur en ${ }^{14} \mathrm{C}$ de l'atmosphère, il est nécessaire de recourir aux collections de coquillages rapportées par les naturalistes avant $1950 \mathrm{AD}$ pour mesurer le $\Delta \mathrm{R}$ actuel. Pour les périodes antérieures la solution consiste à connaître l'âge du niveau archéologique (essentiellement grâce à la typologie céramique) ou sédimentologique (principalement par comptage des sédiments varvés) dans lequel est incluse la coquille à dater.

\section{LES UPWELLINGS ET L'EFFET RÉSERVOIR EN MER D'ARABIE}

L'hydrologie dans le nord-ouest de la mer d'Arabie est contrôlée par les variations climatiques saisonnières liées à la mousson indienne. La formation du courant de Somalie le long de la côte est-africaine est une réponse de la circulation de surface aux inversions des vents de mousson; ce courant transporte vers le nord-est des eaux froides de faible salinité. Le courant de Somalie n'est pas continu le long de la côte, mais comporte des tourbillons qui évoluent avec l'intensité de la mousson du Sud-Ouest ${ }^{6}$. En effet, au déclenchement de la mousson (début mai), le courant côtier est-africain de faible intensité s'intensifie, traverse l'équateur, et forme la

3. STUIVER and BRAZUINAS, 1993.

4. STUIVER and POLACH, 1977.

5. STUIVER et al., 1998 ; HUGHEN et al., 2004.

6. FiEUX et al., 1986 ; PRELl et al., 1990 ; YOU, 1997. branche sud du courant de Somalie. La zone d'upwelling centrée vers $4^{\circ} \mathrm{N}$ apparaît en premier puis, quand la mousson est intense, le grand tourbillon de Somalie se forme entre $5^{\circ}$ et $10^{\circ} \mathrm{N}$. Ce dernier engendre la deuxième zone d'upwelling (de Socotra) centrée autour de $10^{\circ} \mathrm{N}$ ainsi que la troisième vers $20^{\circ} \mathrm{N}$ en bordure de la péninsule Arabique le long des côtes omanaises?

Les déterminations des $\Delta \mathrm{R}$ actuels à l'intérieur d'une large région géographique (fig. 1) ont été effectuées à l'aide de coquilles provenant de collections indiennes, du Museum National d'Histoire naturelle de Paris et du Museum de la Smithsonian Institution (tableau 1). Elles ont permis à Dutta et al. ${ }^{8}$ et Southon et al. ${ }^{9}$ d'établir un bilan global de l'effet réservoir actuel le long des rivages de la mer d'Arabie. Le carottage entrepris au large du Pakistan dans des sédiments varvés a permis de compléter cette série de mesures en datant les foraminifères associés aux varves ${ }^{10}$.

Le $\Delta R$ moyen actuel $(n=10)$ pour le nord-ouest de la mer d'Arabie est donc de $205 \pm 20$ ans $(\sigma / \sqrt{\mathrm{n}})$. Staubwasser $e t$ al. ${ }^{11}$ ont étudié une carotte prélevée au large des côtes pakistanaises $\left(24^{\circ} 36^{\prime} \mathrm{N}, 65^{\circ} 55^{\prime} \mathrm{E}\right)$, composée de sédiments laminés bien préservés. Ils ont daté des foraminifères planctoniques (Globigerinoides sacculifer) associés aux lamines annuelles et déterminent ainsi l'évolution de l'effet réservoir de $3550 \mathrm{cal} \mathrm{BC}$ à $8685 \mathrm{cal} \mathrm{BC}$.

Sur le continent, Uerpmann ${ }^{12}$ publie les premières déterminations de $\Delta \mathrm{R}$ du golfe d'Oman. Il choisit les mangroves de Bandar Khayran et de ar-Ra's al Hamra ainsi que quelques sites anthropiques dispersés dans la région de Sur (fig. 1), tous compris entre 4500 et 6500 BP. Pour les sites de mangrove, il sélectionne Avicennia marina pour la datation du matériau d'origine continental et Terebralia palustris pour celui d'origine marine. La comparaison de ces mesures permet aux auteurs de proposer un effet réservoir d'environ 800 ans $(R+\Delta R)$. Cette valeur, très élevée (tableau 2$)$, met en évidence la difficulté de mesurer avec une grande précision l'effet réservoir en partant de deux mesures ${ }^{14} \mathrm{C}$ croisées. L'expérience acquise dans d'autres zones d'upwelling (e.g. Pérou et Chili) montre que pour certains sites, la matière organique (restes de végétaux, de bois ou de charbons diffus) associée aux coquilles n'a pas toujours un âge négligeable et par
7. PRELL, 1984.
8. DUTTA et al., 2001.
9. SOUTHON et al., 2002.
10. VON RAD et al., 1999.
11. STAUBWASSER et al., 2002.
12. UERPMANN, 1991. 


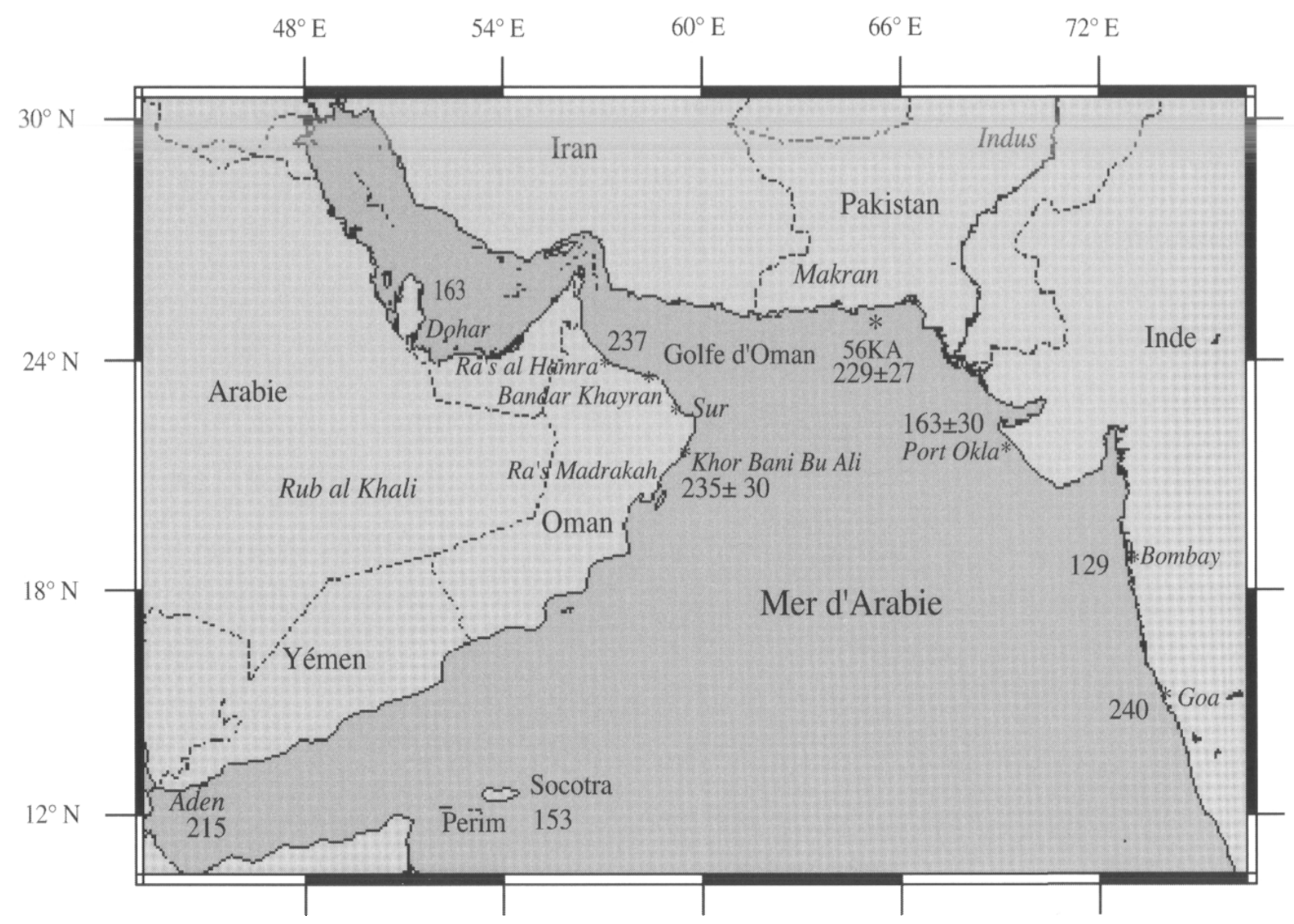

Fig. 1 : $\Delta R$ actuels du bassin de la mer d'Arabie. Lorsque plusieurs mesures ont été effectuées sur un site, nous avons choisi de présenter la moyenne.

ailleurs migre souvent d'une strate à l'autre ; il s'ensuit des erreurs importantes. Biagi ${ }^{13}$ publie une synthèse de dates ${ }^{14} \mathrm{C}$ de coquilles provenant de sites côtiers omanais et se heurte aux mêmes difficultés. Pour les contourner, Charpentier et al. ${ }^{14}$ choisissent un niveau archéologique à Khor Bani Abu 'Ali incluant une céramique datée de $2150 \pm 50 \mathrm{cal} \mathrm{BC}^{15}$ et riche en coquilles d'Amiantis umbonella, et déterminent ainsi un $\Delta \mathrm{R}$ de $235 \pm 30$ ans $^{16}$. Elle montre que l'effet réservoir n'a pas ou peu varié entre l'actuel et le milieu du Ve millénaire (4550 cal BC) et donc que les multiples facteurs qui le contrô-

\section{BIAGI, 1994}

14. ChARPENTIER et al., 2000.

15. MÉRY and MARQUIS, 1998.

16. Cette valeur est cohérente par rapport aux données de VON RAD et al., 1999 ; DUTTA et al., 2001 ; STAUBWASSER et al., 2002 et SOUTHON et al., 2002. lent (mousson d'été, upwelling, mousson d'hiver, surface de convection...) sont restés constants. Le $\Delta \mathrm{R}$ est très mal connu au millénaire précédent (entre $4550 \mathrm{cal} \mathrm{BC}$ et $5500 \mathrm{cal} \mathrm{BC}$ ) et semble fluctuer entre 240 et 610 ans (tableau 2). Ensuite, il se stabilise autour de 610 ans de $5550 \mathrm{cal} \mathrm{BC}$ à $6400 \mathrm{cal} \mathrm{BC}$. La mer a alors atteint son niveau actuel ${ }^{17}$ recouvrant les sites archéologiques antérieurs. À partir de cette période et jusqu'à $8685 \mathrm{cal} \mathrm{BC}$, l'effet réservoir marin devient beaucoup plus important $(R+\Delta R$ de 780 à 1120 ans, tableau 2). Cette évolution de l'effet réservoir est synchrone de l'évolution climatique du nord-ouest de l'océan Indien ${ }^{18}$ qui montre que les flux de la mousson indienne et leurs effets sur l'upwelling de la mer d'Arabie ont considérablement varié.

17. LAMBECK, 1996.

18. SIROCKO et al., 1993 ; METHA and LAU, 1997 ; SCHULZ et al., 1998 ; GUPTA et al., 2003. 
Tableau 1 : Récapitulatif des mesures du $\Delta R$ actuel en mer d'Arabie

\begin{tabular}{|c|c|c|c|c|c|c|}
\hline Espèces & Collection & Date & Localisation & Latitude & Longitude & $\Delta \mathbf{R}$ \\
\hline \multicolumn{7}{|c|}{ Nord-Ouest de la mer d'Arabie } \\
\hline Marcia flammea & MNHN* & $1843 \mathrm{AD}$ & Mascate & $23.5 \mathrm{~N}$ & $58.6 \mathrm{E}$ & 285 \\
\hline Marcia flammea & MNHN* & $1850 \mathrm{AD}$ & Aden & $12.5 \mathrm{~N}$ & $45 \mathrm{E}$ & 157 \\
\hline Gafrarium callipygum & $\mathrm{MNHN}^{*}$ & $1850 \mathrm{AD}$ & Socotra & $12.5 \mathrm{~N}$ & $54 \mathrm{E}$ & 206 \\
\hline Pinctada margaritifera & MNHN* & $1897 \mathrm{AD}$ & Mascate & $23.5 \mathrm{~N}$ & $58.6 \mathrm{E}$ & 189 \\
\hline Irus rugosus & MNHN* & $1920 \mathrm{AD}$ & Perim & $12.5 \mathrm{~N}$ & $53.3 \mathrm{E}$ & 101 \\
\hline Marcia flammea & MNHN* & $1921 \mathrm{AD}$ & Aden & $12.5 \mathrm{~N}$ & $45 \mathrm{E}$ & 287 \\
\hline Pinctada radiata & MNHN* & 1921AD & Aden & $12.5 \mathrm{~N}$ & $45 \mathrm{E}$ & 202 \\
\hline foraminifères & SO90-56KA** & $1926 \mathrm{AD}$ & Carottage & $24^{\circ} 50^{\prime} \mathrm{N}$ & $65^{\circ} 55^{\prime} \mathrm{E}$ & $208 \pm 25$ \\
\hline foraminifères & SO90-56KA** & $1898 \mathrm{AD}$ & Carottage & $24^{\circ} 50^{\prime} \mathrm{N}$ & $65^{\circ} 55^{\prime} \mathrm{E}$ & $248+24$ \\
\hline Turbo brunneus & Inde*** & $1953 \mathrm{AD}$ & Port Okla & $22^{\circ} 28 \mathrm{~N}$ & $69^{\circ} 05^{\prime} \mathrm{E}$ & $163 \pm 30$ \\
\hline \multicolumn{7}{|l|}{ Est de la mer d'Arabie } \\
\hline Marcia recens & MNHN* & $1836 \mathrm{AD}$ & Bombay & $18.8 \mathrm{~N}$ & $72.7 \mathrm{E}$ & 151 \\
\hline gastéropodes & USNM* & $1887 \mathrm{AD}$ & Bombay & $18.9 \mathrm{~N}$ & $72.8 \mathrm{E}$ & 107 \\
\hline Marcia opima & MNHN* & $1841 \mathrm{AD}$ & Goa & $16.5 \mathrm{~N}$ & $73.8 \mathrm{E}$ & 240 \\
\hline \multicolumn{7}{|l|}{ Golfe arabo-persique } \\
\hline Pinctada radiata & MNHN* & $1952 \mathrm{AD}$ & Dohar & $25.3 \mathrm{~N}$ & $51.4 \mathrm{E}$ & 163 \\
\hline \multicolumn{7}{|l|}{ Mer Rouge } \\
\hline Tapes deshayesi & $\mathrm{MNHN}^{*}$ & $1921 \mathrm{AD}$ & Djibouti & $11.3 \mathrm{~N}$ & $43 \mathrm{E}$ & 93 \\
\hline Pinctada radiata & MNHN & $1839 \mathrm{AD}$ & Mer Rouge & 20 & 38 & 96 \\
\hline
\end{tabular}

* MNHN : collection du Museum National d'Histoire naturelle (SOUTHON et al., 2002).

* USNM : Smithsonian Institution (SOUTHON et al., 2002).

** Carotte SO90-56KA (VON RAD et al., 1999).

*** Collection indienne (DUTTA et al., 2001).

\section{CONCLUSION}

La datation ${ }^{14} \mathrm{C}$ des biominéralisations d'origine marine des sites côtiers en mer d'Arabie a été longtemps entachée de l'incertitude sur l'ampleur de l'effet réservoir et son évolution au cours du temps. Nous avons présenté ici la revue des mesures récemment publiées, qui s'appuient sur des référentiels de nature différente (coquilles, comptage des varves et céramiques). Ces données permettent aujourd'hui une bonne estimation du $\Delta \mathrm{R}$, de l'actuel à $8685 \mathrm{cal} \mathrm{BC}$. Du présent à $4560 \mathrm{cal}$ $\mathrm{BC}$, le $\Delta \mathrm{R}(210 \pm 15$ ans, $\mathrm{n}=12)$ pour le nord-ouest de la mer d'Arabie est constant. Il est donc dorénavant possible de calibrer correctement les dates des biominéralisations marines et de les insérer dans les chronologies archéologiques. Au cours de l'Holocène, il varie de façon très importante jusqu'à atteindre 720 ans de 6475 à 7305 cal BC, puis diminue autour d'une valeur de 380 ans entre 7915 et $8685 \mathrm{cal} \mathrm{BC}$.
Cette revue illustre toute l'importance et la difficulté des mesures croisées (sur la matière organique, la céramique, les coquilles marines et le comptage des lamines sédimentaires) pour l'élaboration d'une chronologie fiable des événements « culturels » telles les phases d'occupation humaine le long du littoral et «naturels » telles les fluctuations récurrentes du climat. Dans le nord-ouest de l'océan Indien, les fluctuations du régime des moussons ont entraîné des modifications considérables du milieu océanique au cours de l'Holocène et les remontées d'eau profonde liées aux phases d'augmentation des flux de la mousson d'été ont induit d'importantes variations de la teneur en carbone inorganique dissous utilisé par les organismes vivants pour la formation de leur coquille. La variabilité du climat au cours de l'Holocène est une donnée fondamentale à prendre en compte, pour comprendre l'homme et son histoire. 
Tableau 2 : Bilan des $\Delta R$ de l'actuel à 8685 cal $B C$ dans le nord-ouest de la mer d'Arabie. $L$ 'effet réservoir a été publié sous la forme $\Delta R$ ou $R$. Lorsque $R$ ou $\Delta R$ est suivi de son incertitude, il provient directement des publications, en revanche, précédé de $\sim$, il provient de nos calculs effectués par souci d'homogénéité.

\begin{tabular}{|c|c|c|c|}
\hline Publications & Référentiel $\mathrm{AD}$ ou cal $\mathrm{BC}$ & $\Delta \mathbf{R}$ ans & $\mathbf{R}+\Delta \mathbf{R}$ ans \\
\hline $\begin{array}{l}\text { SOUTHON et al., } 2002 \\
\text { DUTTA et al., } 2001\end{array}$ & $\begin{array}{l}\text { Collections de coquillage }(n=8) \text { : } \\
\text { 1846-1953 AD }\end{array}$ & $200 \pm 20$ & $\sim 605$ \\
\hline VON RAD et al., 1999 & $\begin{array}{l}\text { Sédiments laminés }(n=2) \\
1926 \text { et } 1898 \text { AD }\end{array}$ & $229 \pm 27$ & $\sim 627$ \\
\hline CHARPENTIER et al., 2000 & $\begin{array}{l}\text { Céramique } \\
2150 \pm 50 \text { cal BC }(n=2)\end{array}$ & $235 \pm 30$ & $\sim 635$ \\
\hline UERPMANN, 1991 & $\begin{array}{l}\text { Matière organique continentale }\left({ }^{14} \mathrm{C}\right) \\
3630 \text { à } 4570 \mathrm{cal} \mathrm{BC}(\mathrm{n}=3)\end{array}$ & $\sim 400^{*}$ & $800 *$ \\
\hline STAUBWASSER et al., 2002 & $\begin{array}{l}\text { Sédiments laminés }(\mathrm{n}=12) \\
3550 \text { à } 4560 \mathrm{cal} \mathrm{BC}\end{array}$ & $\sim 240$ & $640 \pm 120$ \\
\hline STAUBWASSER et al., 2002 & $\begin{array}{l}\text { Sédiments laminés }(n=5) \\
4745 \text { à } 5525 \text { cal BC }\end{array}$ & $\sim 425^{* *}$ & $\sim 825^{* *}$ \\
\hline STAUBWASSER et al., 2002 & $\begin{array}{l}\text { Sédiments laminés }(\mathrm{n}=10) \\
5525 \text { à } 6405 \text { cal BC }\end{array}$ & $\sim 610$ & $1010-60 /+140$ \\
\hline STAUBWASSER et al., 2002 & $\begin{array}{l}\text { Carotte de sédiments laminés }(\mathrm{n}=9) \\
6475 \text { à } 7305 \mathrm{cal} \mathrm{BC}\end{array}$ & $\sim 720$ & $1120-70 /+110$ \\
\hline STAUBWASSER et al., 2002 & $\begin{array}{l}\text { Carotte de sédiments laminés }(\mathrm{n}=8) \\
7915 \text { à } 8685 \mathrm{cal} \text { BC }\end{array}$ & $\sim 380$ & $780-80 /+20$ \\
\hline
\end{tabular}

* $\Delta \mathrm{R}$ discordants par rapport à la cohérence des autres mesures ; ** STAUBWASSER et al. soulignent la grande dispersion de ces mesures.

Remerciements : Ce travail a été soutenu par le programme Climat et Environnement d'Arabie depuis le Dernier Maximum Glaciaire, implication pour les populations humaines (programme Eclipse du CNRS).

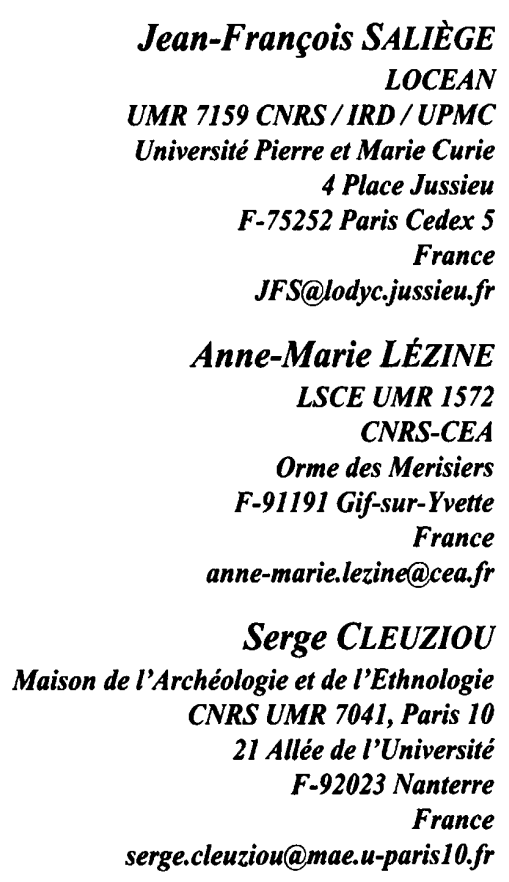

\section{BIBLIOGRAPHIE}

BIAGI P.

1994 A radiocarbon chronology for the aceramic shell-middens of coastal Oman. Arabian archaeology and epigraphy 5 : 1731.

Charpentier V., ANGelucci D., MÉry S. et SALiĖGe J.F.

2000 Autour de la mangrove morte de Suwayh, l'habitat VIc-Ve millénaires de Suwayh SWY-11, Sultanat d'Oman. Proceedings of the Seminar for Arabian Studies 30:69-85.

ClEuZiou S. and TOSI M.

1989 The southeastern Frontier of the Ancient Near East. In : FRIFELT K. and SORENSEN P. (eds), South Asian Archaeology 1985 : 15-47. London : Curzon press.

DUTTA K., BHUSHAN K. and SOMAYAJULU B.L.K.

$2001 \Delta \mathrm{R}$ correction values for the northen Indian ocean. Radiocarbon $43,2: 483-488$.

FIEUX M., SCHOTT F. and SWALLOW J.C.

1986 Deep boundary currents in the western Indian Ocean revisited. Deep-sea Res. 33,4 : 415-426.

GUPTA A.K., ANDERSON D.M. and OVERPECK J.T.

2003 Abrupt changes in the Asian southwest monsoon during the Holocene and their links to the north Atlantic Ocean. Nature $421: 354-357$. 
Hughen K.A., BAILlie M.G.L., BAYLISS A., BeCK J.W., BERTRAND C., BLACKWELL P., BUCK C.E., BURR G., CUTLER K.B., DAMON P.E., EDWARDS R.L., FAIRBANKS R.G., FRIEDRICH M., GUILDERSON T.P., KROMER B., MCCORMAC F.G., MANNIN S., BRONK RAMSEY C., REIMER P.J., REIMER R.W., REMMELE S., SOUTHON J., STUIVER J.R., TALAMO S., TAYLOR F.W., VAN DER PLICHT J. and WEYHEMMEYER C.E.

2004 Marine data. Radiocarbon $46:$ 1059-1086.

LAMBECK K.

1996 Shoreline reconstruction for the Persian Gulf since the last glacial maximum. Earth and Planetary Science Letters 142 : 43-57.

MÉRY S.

1991 Origine et production des récipients de terre cuite dans la péninsule d'Oman à l'âge du Bronze. Paléorient 17,2: 5178.

1996 Ceramics and patterns of exchange across the Arabian sea and the Persian gulf in the early Bronze age. I $n$ : AFANAS'E G., CLEUZIOU S., LUKACKS J.R. and TOSI M. (eds), The Prehistory of Asia and Oceania. Forli, 8-14 septembre, 1996, XIII' International Congress of Prehistoric and Protohistoric Sciences $16: 167-179$. Forli : ABACO.

MÉRY S. and MARQUIS P.

1998 First campaign of excavation at Khor Bani Bu Ali SWY3 Sultanate of Oman. Proceedings of the Seminar for Arabian Studies $28: 215-218$.

METHA V. and LAU K.-M.

1997 Influence of the solar irradiance on the Indian monsoonENSO relationship at decadal-multidecadal time scales. $\mathrm{Geo}$ phys. Res. Lett. 24 : 159-162.

PRELL W.L.

1984 Monsoonal climate of the Arabian Sea during the late Quaternary : a reponse to changing solar radiation. In : BERGER A., IMBRIE J. and HAYS J. (eds), Milankovitch and climate, Part 1: 349-366. Dordrecht, Boston, Lancaster: D. Reidel Publishing Company.

PRELL W.L. and MARVIL E.R.

1990 Variability in upwelling fields in the northwestern Indian Ocean. Paleoceanography 12,3:447-457.
SCHULZ H., von RAD U. and ERLENKEUSER H.

1998 Correlation between Arabian Sea and Greenland climate oscillations of the past 110000 years. Nature $393: 54-57$.

SiRoCKo F., SARTHIEN M., ERLENKEUSER H., LANGE H., ARNOLD M. and DUPLESSY J.-C.

1993 Century-scale events in monsoonal climate over the past 24000 years. Nature $364: 322-324$.

SOUTHON J., KASHGARIAN M., FONTUGNE M., METIVIER B. and YIM W.

2002 Marine reservoir corrections for the Indian ocean and southeast Asia. Radiocarbon 44,1 : 167-180.

STAUBWASSER M., SiRocKo F., GROOTES P. and ERLENKEUSER H.

2002 South Asian monsoon climate change and radiocarbon in the Arabian Sea during early and middle Holocene. Paleoceanography $17,4: 1063$.

STUIVER M. and POLACH H.A.

1977 Discussion reporting of ${ }^{14} \mathrm{C}$ data. Radiocarbon $19,3: 355$ 363.

STUIVER M. and BRAZIUNAS T.

1993 Modeling atmospheric influences and ${ }^{14} \mathrm{C}$ ages of marine samples to 10000 BC. Radiocarbon 35,1: 137-189.

STUIVER M., REIMER P.J. and BRAZIUNAS T.F.

1998 High-precision radiocarbon age calibration for the terrestrial and marine samples. Radiocarbon $40,3: 1127-1151$.

UERPMANN H.-P.

1991 Radiocarbon dating of shell middens in the Sultanate of Oman. PACT 29 : 335-347.

VON RAD U., SCHAFF M., MICHELS K.H., BERGER W.H. and SIROCKO F.

1999 A 5000 -yr record of climatic change in varved sediments from the oxygen minimum zone of Pakistan, northeastern Arabian sea. Quaternary Research $51: 39-53$.

YOU Y.

1997 Seasonal variations of thermocline circulation and ventilation in the Indian Ocean. Journal of Geophysical Research $102: 10391-422$. 